\title{
Atomic charge exchange between semi-relativistic helium ions and targets from carbon to lead.
}

\author{
R. Meharchand ${ }^{1,2,3 *}$, H. Akimune ${ }^{4}$, A.M. van den Berg, ${ }^{5}$, Y. Fujita ${ }^{6}$, M. Fujiwara $^{7,8}$, S. \\ Galés $^{9}$, M.N. Harakeh ${ }^{5}$, H. Hashimoto ${ }^{8}$, R. Hayami ${ }^{10}$, G.W. Hitt ${ }^{1,2,3}$, M. Itoh ${ }^{11}$, T. \\ Kawabata $^{12}$, K. Kawase ${ }^{7}$, M. Kinoshita ${ }^{4}$, K. Nakanishi ${ }^{13}$, S. Nakayama ${ }^{10}$, S. \\ Okumura $^{8}$, Y. Shimbara ${ }^{14}$, M. Uchida ${ }^{15}$, T. Yamagata ${ }^{4}$, M. Yosoi ${ }^{8}$, R.G.T. Zegers ${ }^{1,2,3}$ \\ ${ }^{1}$ National Superconducting Cyclotron Laboratory, Michigan State University, East Lansing, MI 48824-1321, USA \\ ${ }^{2}$ Department of Physics and Astronomy, Michigan State University, East Lansing, MI 48824, USA \\ ${ }^{3}$ Joint Institute for Nuclear Astrophysics, Michigan State University, East Lansing, MI 48824, USA \\ ${ }^{4}$ Department of Physics, Konan University, Kobe, Hyogo, 658-8501, Japan \\ ${ }^{5}$ Kernfysisch Versneller Instituut, University of Groningen, Zernikelaan 25, 9747 AA Groningen, The Netherlands \\ ${ }^{6}$ Department of Physics, Osaka University, Toyonaka, Osaka 560-0043, Japan \\ ${ }^{7}$ Kansai Photon Science Institute, Japan Atomic Energy Agency, Kizu, Kyoto 619-0215, Japan \\ ${ }^{8}$ Research Center for Nuclear Physics, Osaka University, Ibaraki, Osaka 567-0047,Japan \\ ${ }^{9}$ Institut de Physique Nucléaire, IN2P3-CNRS, Orsay, France \\ ${ }^{10}$ Department of Physics, University of Tokushima, Tokushima 770-8502, Japan \\ ${ }^{11}$ Cyclotron and Radioisotope Center, Tohoku University, Sendai, Miyagi 980-8578, Japan \\ ${ }^{12}$ Center for Nuclear Study, University of Tokyo, RIKEN Campus, Wako, Saitama 351-0198, Japan \\ ${ }^{13}$ Center for Nuclear Study, University of Tokyo, Bunkyo, Tokyo 113-0033, Japan \\ ${ }^{14}$ Graduate School of Science and Technology, Niigata University 8050 Ikarashi 2-nocho, Nishi-ku, Niigata 950-2181 \\ ${ }^{15}$ Tokyo Institute of Technology, 2-12-1 O-Okayama, Tokyo 152-8550, Japan \\ E-mail: zegers@nscl.msu.edu
}

In $\left({ }^{3} \mathrm{He}, t\right)$ nuclear charge-exchange reactions, ${ }^{3} \mathrm{He}^{+}$charge states are produced as a result of atomic charge-exchange between ${ }^{3} \mathrm{He}^{++}$ions and atoms in the target. In equilibrium, the atomic charge-exchange process consists of both electron ionization (stripping), and electron capture, which can proceed through Radiative Electron Capture (REC) or Non-Radiative Electron Capture (NREC). Unfortunately, a complete description of the atomic charge-exchange process is lacking. As a result, a largely phenomenological approach has been used, which has not been tested for high beam energies. In this work, ratios of equilibrium charge-state yields for singly to doubly ionized ${ }^{3} \mathrm{He}$ ions were measured using the Grand Raiden Spectrometer at the Research Center for Nuclear Physics (RCNP). A semi-phenomenological approach used when calculating atomic electron capture and stripping cross sections is successful in describing the data for beam energies of $\mathrm{E}\left({ }^{3} \mathrm{He}\right)=420 \mathrm{MeV}$.

10th Symposium on Nuclei in the Cosmos

July 27 - August 1, 2008

Mackinac Island, Michigan, USA

*Speaker.

${ }^{\dagger}$ We thank the cyclotron staff at RCNP for their support during the experiment. This work was supported by the US NSF (PHY-0606007), the Ministry of Education, Science, Sports and Culture of Japan, the Stichting voor Fundamenteel Onderzoek der Materie (FOM), the Netherlands. 


\section{Introduction}

The $\left({ }^{3} \mathrm{He}, t\right)$ nuclear charge-exchange reaction has been widely used to study the spin-isospin response of nuclei, in particular Gamow-Teller transitions which are important to extract weak transition strengths in excitation-energy regions inaccessible to $\beta$-decay [1]. Recently, these experiments have been performed at RCNP with ${ }^{3} \mathrm{He}^{++}$beams of $\mathrm{E}\left({ }^{3} \mathrm{He}\right)=420$ and $450 \mathrm{MeV}$. Along with the scattered tritons, ${ }^{3} \mathrm{He}^{+}$ions originating from atomic charge-exchange reactions produce a strong peak in the focal plane of the spectrometer Grand Raiden [2]. These ${ }^{3} \mathrm{He}^{+}$charge-state contaminants are useful for calibration purposes, as they provide information about the angular spread of the incoming beam and designate the central beam axis.

The present work is motivated by the potential for the use of ${ }^{3} \mathrm{He}^{+}$atomic charge-exchange events to be used in normalization procedures for $\left({ }^{3} \mathrm{He}, t\right)$ nuclear charge-exchange experiments. Other applications include using REC photons as an observable for ${ }^{3} \mathrm{He}^{++}$ions in extragalactic space [3] and estimating charge-state production for the design of future radioactive ion beam facilities.

No comprehensive theory exists describing the atomic charge-exchange process over a wide energy and mass range. A semi-phenomenological approach is often used, based on the works of Allison [4], Betz [5], Bohr [6], Gillespie [7] and Nikolaev [8] and tested experimentally by Katayama et al. [9], Dennis et al. [10] and Gójska et al. [11]. This approach takes into account the balance between electron stripping and capture contributions, and the results of the calculations are in good agreement with data obtained over the energy range tested thus far $\left(\mathrm{E}\left({ }^{3} \mathrm{He}\right)=67.9 \mathrm{MeV}\right.$, 99.2 MeV, 130.2 MeV [9], and $200 \mathrm{MeV}$ [10]).

We studied the atomic charge-exchange between ${ }^{3} \mathrm{He}$ ions at $420 \mathrm{MeV}(\beta=0.49)$ and a variety of targets with different atomic number. The experimental results are compared with the theoretical calculations mentioned above and are an extension to higher beam energies of the work by Dennis et al. [10]. We also compare results with the predictions of the codes CHARGE and GLOBAL [12] (implemented in the code LISE++ [13]), to demonstrate the need for improved estimates of charge-exchange cross sections for fast radioactive ion beam experiments.

\section{Experiment}

The details of the experimental setup are the same as given in Ref. [1]. An incident beam of ${ }^{3} \mathrm{He}^{++}$particles with a kinetic energy $\mathrm{E}=420 \mathrm{MeV}$ was generated at the Ring Cyclotron Facility at RCNP with beam intensities between 4 and $10 \mathrm{enA}$. This beam was used to bombard isotopicallyenriched targets of ${ }^{12} \mathrm{C},{ }^{26} \mathrm{Mg},{ }^{60} \mathrm{Ni},{ }^{90} \mathrm{Zr},{ }^{120} \mathrm{Sn}$, and ${ }^{208} \mathrm{~Pb}$. All targets were sufficiently thick to reach equilibrium between electron stripping and capture and thus the charge-state distribution was independent of target thickness.

The ${ }^{3} \mathrm{He}^{+}$ions were detected at the focal plane of the spectrometer, along with the tritons produced in the nuclear charge-exchange reaction. The two products were easily separated using energy-loss measurements through a stack of focal plane scintillators. For each target, the number of ${ }^{3} \mathrm{He}^{+}$charge-state events was summed and the total yield was corrected for data-acquisition dead time $(\sim 1 \%) .{ }^{3} \mathrm{He}^{++}$ions were collected in a Faraday cup placed in the first dipole magnet of the spectrometer. The systematic error in current integration was estimated to be less than $10 \%$, and is 
the same for all targets used. Since this would only lead to an overall scaling factor, the systematic error is not indicated in the included figure.

\section{Theoretical Cross-Section Estimates}

The charge-state distribution following atomic charge-exchange reactions depends strongly upon the velocity of the incoming particles and the atomic number of the target atoms. These factors are included in the descriptions of capture and stripping cross sections. In the case of equilibrium charge-state distribution, there is a simple relationship connecting the charge-state yield ratios to the capture and stripping cross-sections $[4,5]$ :

$$
\frac{Y\left({ }^{3} H e^{+}\right)}{Y\left({ }^{3} H e^{++}\right)}=\frac{\sigma_{c a p}}{\sigma_{\text {strip }}} .
$$

By measuring the charge-state distributions, the ratio of theoretical estimates for the capture and stripping cross sections can thus be tested.

Nikolaev calculated atomic capture cross sections for protons colliding with multi-electron atoms using hydrogen-like wave functions in the one-electron variant of the Brinkmann-Kramers' approximation [8]. The cross section for the capture of an electron into a projectile state with principal quantum number $n_{a}$ from a target with a fully-filled electron shell of principal quantum number $n$ is determined by:

$$
\sigma_{c a p}\left(n_{a} \mid n\right)=\pi a_{0}^{2} \frac{2^{8}}{5} N_{a} n^{2}\left(\frac{v_{0}}{v}\right)^{2} \gamma^{5} \eta_{n}^{5}(1+\beta)^{\frac{5}{2}}(1+\beta \gamma)^{-3} \Phi_{4}(\beta \gamma),
$$

where $a_{0} \simeq 5.292 \times 10^{-9} \mathrm{~cm}$ and $v_{0} \simeq 2.188 \times 10^{8} \mathrm{~cm} / \mathrm{s}$ are the atomic units of length (Bohr radius) and velocity, respectively and $N_{a}$ is the number of electrons in the shell with principal quantum number $n_{a}$. The other parameters in Eq. 3.2 are defined as: $\gamma=4 V^{-2}\left[1+2\left(1+\eta_{n}^{2}\right) V^{-2}+(1-\right.$ $\left.\left.\eta_{n}^{2}\right)^{2} V^{-4}\right]^{-1}, V=v / u$ with $u=\left(2 \varepsilon_{a} / \mu\right)^{1 / 2}, \eta_{n}=Z v_{0} / n u$ and $\beta=\mu v_{0}^{2} b_{a}^{2} /\left(2 \varepsilon_{a} c^{2}\right)-1$. In these equations, $v$ is the speed of the projectile (in $\mathrm{cm} / \mathrm{s}$ ), and $\varepsilon_{a}$ is the weighted average of the binding energies of electrons in $\mathrm{keV} . \mu$ is the mass of the electron $(\mathrm{keV}), Z$ is the charge of the projectile $\left(Z=2\right.$ for ${ }^{3} \mathrm{He}$ ), and $b_{a}$ describes the screening effect due to other electrons in the target atom. To calculate $b_{a}$, the effective charge of the nucleus is divided by the principal quantum number $n_{a}$ of the electron shell from which capture occurs. The effective charge $Z_{T}^{*}$ equals $Z_{T}-s$, where $\mathrm{s}$ is calculated using the Slater rules [14]. Finally, $\Phi_{4}$ was approximated as $\Phi_{4}=1-0.25 \beta \gamma$, which is valid for $\beta \gamma<1$ [8], as is the case here .

In Ref. [8] Eq. (3.2) was renormalized to ensure agreement with experimental capture cross sections for protons at low energies. This phenomenological renormalization was introduced as:

$$
R_{0}(t)=\frac{0.3}{\left(t^{-8}+t\right)^{0.2}}, t=\frac{7}{9} \frac{v}{v_{0} \sqrt{b_{a}}} .
$$

Further discussion of this correction is given in Ref. [10].

Classical approximations for the stripping cross section of low, medium, and high- $Z_{T}$ targets were derived by Bohr [6]. For the medium- $Z_{T}$ case the stripping cross section is given by:

$$
\sigma_{\text {strip }}=\pi a_{0}^{2} \frac{Z_{T}^{2 / 3}}{Z}\left(\frac{v_{0}}{v}\right) \text {. }
$$




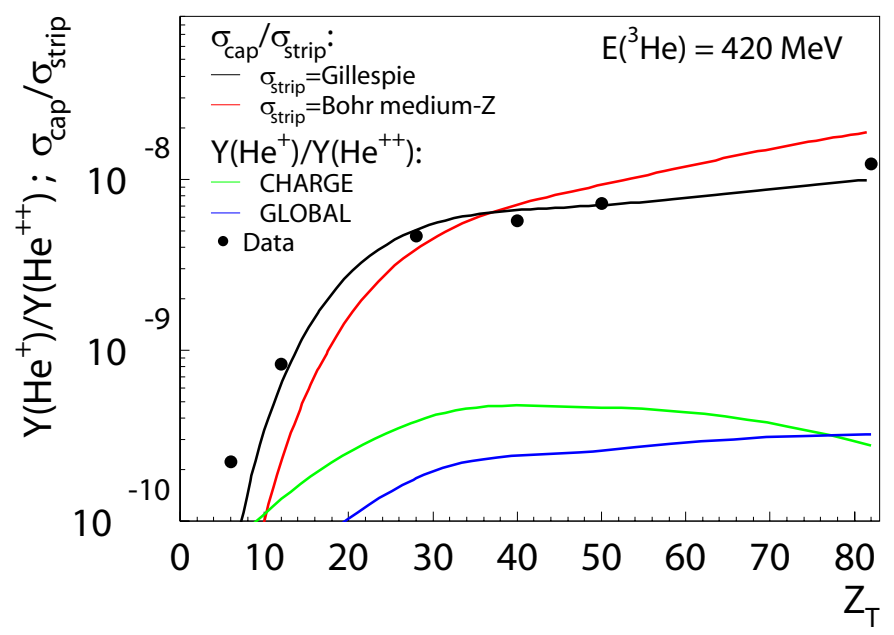

Figure 1: $\mathrm{Y}\left({ }^{3} \mathrm{He}^{+}\right) / \mathrm{Y}\left({ }^{3} \mathrm{He}^{++}\right)$yield ratios obtained at $\mathrm{E}\left({ }^{3} \mathrm{He}\right)=420 \mathrm{MeV}$ and comparison to calculated ratios $\sigma_{\text {cap }} / \sigma_{\text {strip }}$. The black points correspond to our measured values. Statistical errors are negligibly small. The black and red lines correspond to calculated cross-section ratios using the Gillespie and Bohr stripping cross sections respectively. The green and blue lines correspond to the yield ratios given by the CHARGE and GLOBAL codes, respectively.

An independent description, was given by Gillespie [7] on the basis of the asymptotic (high-energy) Born approximation:

$$
\sigma_{\text {strip }}=8 \pi a_{0}^{2} I_{g}\left(\frac{v_{0}}{v}\right)^{2}
$$

where $I_{g}$ is a purely phenomenological expression for the ionization collision strength (for details, see Ref. [10]):

$$
I_{g}=\frac{1.24}{Z^{2}} Z_{T}\left(1+.105 Z_{T}-5.4 \times 10^{-4} Z_{T}^{2}\right) .
$$

It was previously found that at energies up to $200 \mathrm{MeV}[9,10]$ a combination of the descriptions by Bohr for medium- $Z$ targets and Gillespie reproduced the data if the latter approach was used for low-Z nuclei. We have found that at $420 \mathrm{MeV}$, the Gillespie approximation represents the data over the full target range.

\section{Comparison Between Experimental Results and Theory}

In Fig. 1, the measured charge-state yield ratios are compared with the calculated capture-tostripping cross-section ratios as a function of atomic number of the target. Eq. (3.2) was employed in the calculation of the capture cross section, and Eqs. (3.4-3.6) were used in the stripping crosssection calculations. In contrast to the studies performed at lower beam energies, we find that using only the stripping cross-section calculation by Gillespie [7] in the calculation of the ratio, gives the best description of the data. This is likely due to the high beam energy resulting in a short passage time of the projectile through the target atom and reducing the contribution from multi-step processes.

Yield ratios calculated using the CHARGE and GLOBAL programs of LISE++ are also included in Fig. 1. As these programs were initially designed for high-energy (80-1000 MeV/u), 
high- $Z$ ( $>29$ ) projectiles [12], it is perhaps not surprising that they fail to reproduce the data taken with a low- $Z$ projectile. Clearly, caution should be taken when using these codes to calculate charge-state distributions for low- $Z$ projectiles, especially as $Z_{T}$ increases.

\section{Conclusion}

The ${ }^{3} \mathrm{He}^{+}$to ${ }^{3} \mathrm{He}^{++}$equilibrium charge-state yield ratios were measured at $\mathrm{E}\left({ }^{3} \mathrm{He}^{++}\right)=420$ $\mathrm{MeV}$ for a variety of targets. The data were compared to the theoretical ratios of electron-capture to stripping cross sections. Except for the case of the ${ }^{12} \mathrm{C}$ target, they were found to be in good agreement. Although these calculations were originally developed for low beam energies, it is found that they work well even beyond the range previously covered (up to $200 \mathrm{MeV}$ ). However, in contrast to the measurements performed at lower energies, there is no need at $\mathrm{E}\left({ }^{3} \mathrm{He}^{++}\right)=$ $420 \mathrm{MeV}$ to apply Bohr's medium- $Z_{T}$ description for stripping cross sections for targets of higher atomic number. Instead, the description by Gillespie works well over the whole $Z_{T}$ range studied here. The applicability of the code CHARGE was tested for beams of light ions. The CHARGE calculations underestimate the experimental yield ratios of ${ }^{3} \mathrm{He}^{+}$to ${ }^{3} \mathrm{He}^{++}$. The acquired data can provide additional testing ground for the development of more rigorous theoretical approaches [11] than applied here.

\section{References}

[1] R.G.T. Zegers et al., Phys. Rev. Lett. 99 (2007) 202501.

[2] M. Fujiwara et al., Nucl. Instr. Meth. Phys. Res. A 422 (1999) 484.

[3] Z. Sujkowski, Nucl. Phys. A 719 (2003) 266c.

[4] S.K. Allison, Rev. Mod. Phys. 30 (1958) 1137.

[5] H.D. Betz, Rev. Mod. Phys. 44 (1972) 465.

[6] N. Bohr, K. Dan. Vidensk. Selsk. Mat.-Fys. Medd. 18 (1948) No. 8.

[7] G.H. Gillespie, Phys. Rev. A 18 (1978) 1967; A 26 (1982) 2421.

[8] V.S. Nikolaev, Sov. Phys. JETP 24 (1967) 847.

[9] I. Katayama et al., Z. Phys. D 3 (1986) 73, and references therein.

[10] K. Dennis et al., Phys. Rev. A 50 (1994) 3992; J. Jänecke, private communication.

[11] A. Gójska et al., Nucl. Instr. and Meth. Phys. Res. B 235 (2005) 368; A. Gójska et al., Eur. Phys. J. A 27 (2006) 181.

[12] C. Scheidenberger et al., Nucl. Instr. Meth. Phys. Res. B 142 (1998) 441.

[13] D. Bazin et al., Nucl. Instr. Meth. Phys. Res. A 482 (2002) 307; O.B. Tarasov, private communication. 1996), F-37.

[14] J.C. Slater, Phys. Rev. 34 (1929) 1293.

[15] A. Gójska, private communication. 